\title{
Transduodenal EUS FNA Diagnosis of Idiopathic Retroperitoneal
}

\section{Fibrosis}

\author{
Romeo Ioan Chira ${ }^{1,2}$, Irina Dragomir ${ }^{2}$, Vlad Andrei Ichim ${ }^{1,2}$, Vasile Virgil Bințințan ${ }^{1,3}$
}

1) Iuliu Hațieganu, University of Medicine and Pharmacy Cluj-Napoca; 2) 1st Medical Clinic, Gastroenterology Department, Emergency Clinical County Hospital Cluj, Cluj-Napoca; 3) 1st Surgical Clinic, Emergency Clinical County Hospital Cluj, Cluj-Napoca, Romania
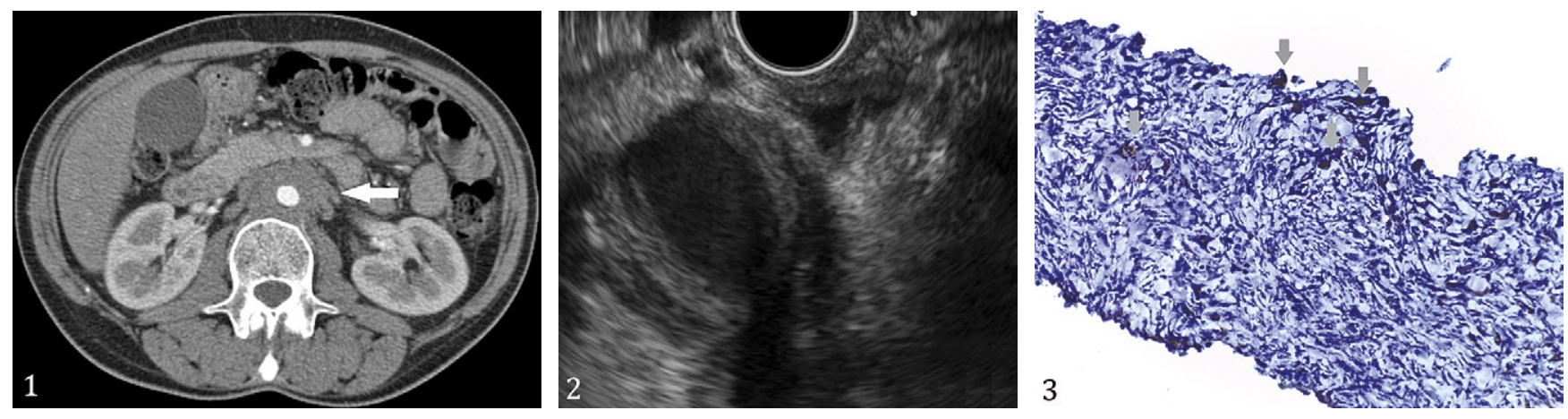

Idiopathic retroperitoneal fibrosis is a rare disease associated with autoimmune inflammatory or sclerosing disorders [1]. It is usually diagnosed by surgical or CT-guided biopsy. We report a case where distal transduodenal endosonography and fine needle aspiration (EUS-FNA) played a central role in the diagnosis of idiopathic retroperitoneal fibrosis.

A 38-year old female patient, known to have ankylosing spondylitis was referred to our department for persistent epigastric pain. A previous abdominal CT scan revealed a retroperitoneal mass surrounding the infrarenal aorta and the first centimeters of iliac arteries (Fig. 1, arrow). The presumed diagnosis was retroperitoneal fibrosis in a patient with another idiopathic inflammatory disease. We decided to perform EUS in order to assess the feasibility of a transduodenal FNA of the mass surrounding the aorta. We advanced the Olympus GF-UCT 180 linear echoendoscope to the 3rd part of the duodenum and visualized a hypoechoic pseudoenlargement of the abdominal aorta wall (Fig. 2). Using color Doppler mode. we chose the appropriate puncture tract and performed, with a 22 gauge needle, two passages in the lesion, "tangent" to the aortic wall. The obtained specimen contained inflammatory cells and had a benign aspect. As there are no literature data about this diagnostic approach, we recommended a laparoscopic-guided biopsy with Tru-cut needle in order to confirm the diagnosis.

The patient was referred to the Surgical Department, where the laparoscopic US-guided biopsy was performed. Histology showed immunohistochemistry staining positive for anti CD45 (Fig. 3, 20x) and an inflammatory infiltrate inside fibrous proliferation, with predominantly diffuse pattern and focal aggregates (arrows), suggestive for idiopathic retroperitoneal fibrosis. We referred the patient back to the rheumatologist for corticotherapy.
Retroperitoneal fibrosis is more frequently associated with autoimmune thyroid diseases, rheumatoid arthritis, ankylosing spondylitis, ANCA-associated vasculitis, systemic lupus erythematosus and psoriasis [2], but it can also be associated with fibro-inflammatory conditions involving other structures [3]. Imaging techniques, such as CT and magnetic resonance imaging are the modalities of choice for diagnosis, but the most reliable method remains a histological examination. Classic biopsy approaches include open surgery or laparoscopy. More recently, retroperitoneoscopic [3] and CT-guided biopsies using FNA needles were described [4]. We used, to the best of our knowledge for the first time, EUS-FNA for diagnosing idiopathic retroperitoneal fibrosis, as a new minimally invasive diagnostic method.

Corresponding author: Romeo Ioan Chira, romeochira@yahoo.com

Conflicts of interest: None to declare.

\section{REFERENCES}

1. van Bommel EF, Jansen I, Hendriksz TR, Aarnoudse AL. Idiopathic retroperitoneal fibrosis: prospective evaluation of incidence and clinicoradiologic presentation. Medicine (Baltimore) 2009;88:193-201. doi:10.1097/MD.0b013e3181afc420

2. Vaglio A, Maritati F. Idiopathic retroperitoneal fibrosis. J Am Soc Nephrol 2016;27:1880-1889. doi:10.1681/ASN.2015101110

3. Miyake M, Fukui S, Gotoh D, et al. The diagnostic utility of retroperitoneoscopic tissue biopsy for unresectable retroperitoneal lesions excluding urogenital cancers. World J Surg Oncol 2019;17:35 doi:10.1186/s12957-019-1581-0

4. Stein AL, Bardawil RG, Silverman SG, Cibas ES. Fine needle aspiration biopsy of idiopathic retroperitoneal fibrosis. Acta Cytol 1997;41:461466. doi:10.1159/000332539 